\title{
Esthetic Management of Dental Fluorosis with Ceramic Veneers
}

\author{
${ }^{1}$ Karishma Jagadeesh, ${ }^{2}$ MN Hombesh, ${ }^{3}$ HS Shashidar, ${ }^{4}$ G Vinay Kumar, ${ }^{5}$ Aditya Sharma, ${ }^{6}$ CR Vijayalakshmi
}

\begin{abstract}
The fluoride may be beneficial as well as harmful to teeth. The effects of fluorosis on teeth range from white opaque areas to severe pitting with brown or black discoloration of the teeth. Such discoloration of teeth causes unesthetic appearance of teeth and requires rehabilitation. The present article describes the case report of esthetic rehabilitation of dental fluorosis with ceramic veneers.
\end{abstract}

Keywords: Dental fluorosis, Laminates, Veneers.

How to cite this article: Jagadeesh $\mathrm{K}$, Hombesh MN, Shashidar HS, Kumar GV, Sharma A, Vijayalakshmi CR. Esthetic Management of Dental Fluorosis with Ceramic Veneers. J Health Sci Res 2018;9(1):27-30.

\section{Source of support: Nil}

Conflict of interest: None

\section{INTRODUCTION}

It is well documented that fluoride can have both beneficial and detrimental effects on the dentition ever since Mc-Kay and Black ${ }^{1}$ published the effect of fluoride on dentition. The beneficial effects of fluoride on dental caries are due to the topical effect of fluoride after the teeth have erupted in the oral cavity. In contrast, detrimental effects are due to systemic absorption of fluoride during tooth development resulting in dental fluorosis. ${ }^{2}$ Fluorosis disturbs enamel significantly and affects esthetics resulting in psychological distress to the affected person. ${ }^{3}$

The clinical manifestations of dental fluorosis may be widely diverse, varying from (i) changes in enamel seen as white flecks, to (ii) mild changes and white opaque areas involving more of the tooth surface, to (iii) moderate and severe changes in tooth tissue seen as pitting and mottling of the surfaces, with or without brown to black staining, to even (iv) severe corrosion of tooth surfaces. ${ }^{4,5}$ Selecting between noninvasive, minimally invasive, and invasive restorative procedures to esthetically treat such

\footnotetext{
${ }^{1,5,6}$ Postgraduate Student, ${ }^{2,3}$ Professor, ${ }^{4} \mathrm{Head}$

${ }^{1-6}$ Department of Prosthodontics, College of Dental Sciences Davangere, Karnataka, India

Corresponding Author: Hombesh MN, Professor, Department of Prosthodontics, College of Dental Sciences, Davangere Karnataka, India, Phone: +919448533612, e-mail: dr_hombesh @yahoo.com
}

fluorosis affected teeth poses a significant challenge to the restorative dentist. ${ }^{6}$ An understanding of all available treatment modalities is vital to the dentist's approach. ${ }^{7}$ Depending upon the severity, treatment option varies from micro/macroabrasion to bleaching, composite restorations, veneers to full coverage restorations. ${ }^{8}$

In India, fluorosis was identified in 1937 in Nellore of Andhra Pradesh by Shortt ${ }^{9}$ Geological crust of India, especially South India, has fluoride-rich minerals which can contaminate underground aquifers. ${ }^{10}$ The Bureau of Indian Standards (BIS) has set the maximum permissible level of fluoride in drinking water at 1 ppm. However, in India, there are areas in which the water may contain fluoride levels in the range of 0.5 to $29 \mathrm{ppm}$. A study where the prevalence and severity of dental fluorosis in rural areas of Davangere, India, was assessed showed fluoride concentration in drinking water was in the range of 0.22 to $3.41 \mathrm{ppm}$. It stated that dental fluorosis is a major dental public health problem among children in Davangere. ${ }^{11}$

\section{CASE REPORT}

\section{Case History, Diagnosis, and Treatment Planning}

A 32-year-old male patient reported to the Department of Prosthodontics with the chief complaint of discolored teeth. A detailed dental and medical history revealed the presence of discoloration since childhood and similar changes within family members. On clinical examination, patient had generalized enamel fluorosis that had affected all of the permanent teeth with the exception of maxillary first molars. Most of the surfaces of the teeth were affected with brownish discoloration (Figs 1 and 2). The appearance ranged from dark brown to black discoloration without pitting. Class 1 occlusal relationship was present with good oral hygiene, and periodontal health was within adequate requirement. Radiographic examination showed absence of caries and alveolar bone loss. Based on the history, clinical findings, and Dean's index, a diagnosis of moderate dental fluorosis was made. Rehabilitation of occlusal surfaces was not required and the patient's main concern was to rehabilitate his present smile. All possible treatment modalities were discussed with the patient. Finally, a restoration that can esthetically mask the stains with minimal loss of tooth structure was reached by opting for treatment with porcelain laminates. 


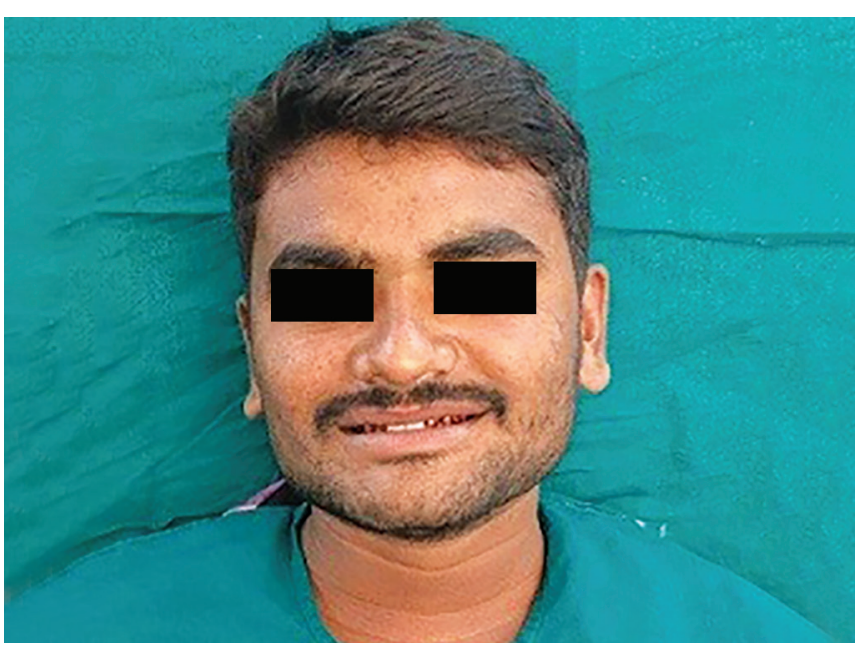

Fig. 1: Pretreatment frontal view

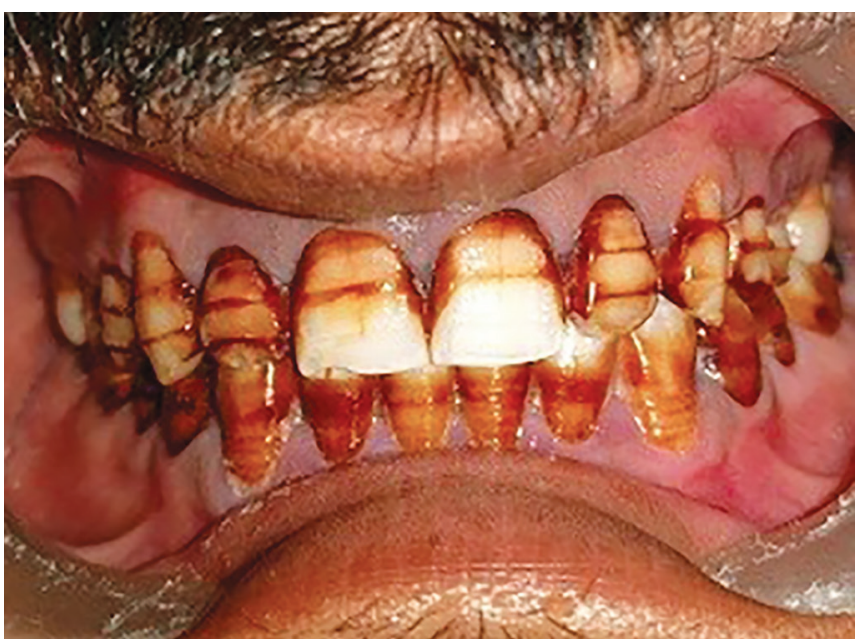

Fig. 3: Depth orientation grooves

Initial Phase (Smile Analysis, Diagnostic Wax-up)

The first phase after the oral prophylaxis included smile analysis and shade selection. Photographs were also taken and study models were made to evaluate the occlusion. Based on the patient's smile line, a decision was made to include maxillary 10 teeth and mandibular 8 teeth in the treatment. Diagnostic mock-up was done and showed to the patient. It was decided to complete maxillary anterior teeth first followed by mandibular anterior teeth. Once the patient was satisfied, phase two was initiated.

\section{Second Phase (Teeth Preparation and Impression)}

The planned teeth were desensitized and three depth orientation grooves were placed on the cervical, middle, and incisal third of the labial surface using depth cutting burs (LVS2). These burs have been specifically designed so that the reduction in the cervical third is shallowest where the enamel thickness is thin. Depth orientation

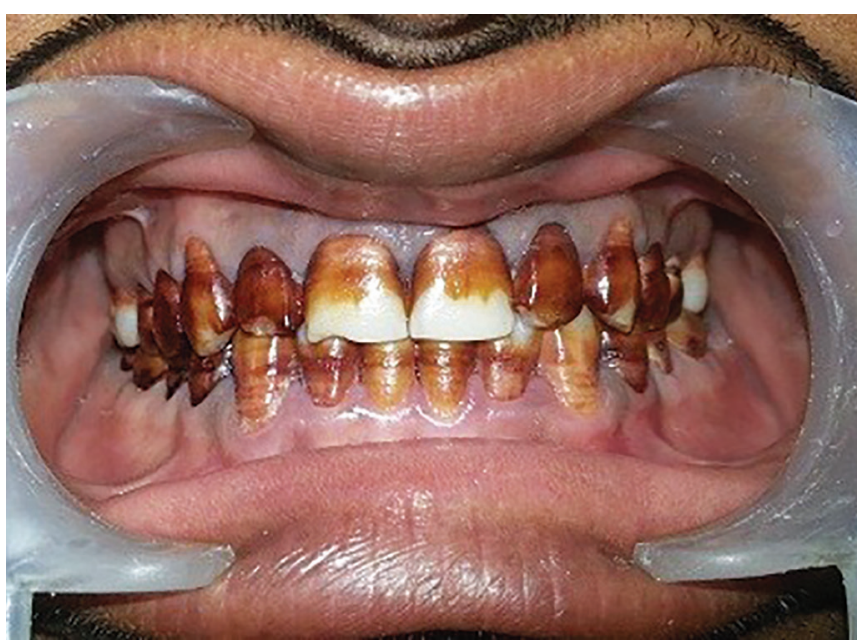

Fig. 2: Pretreatment intraoral view

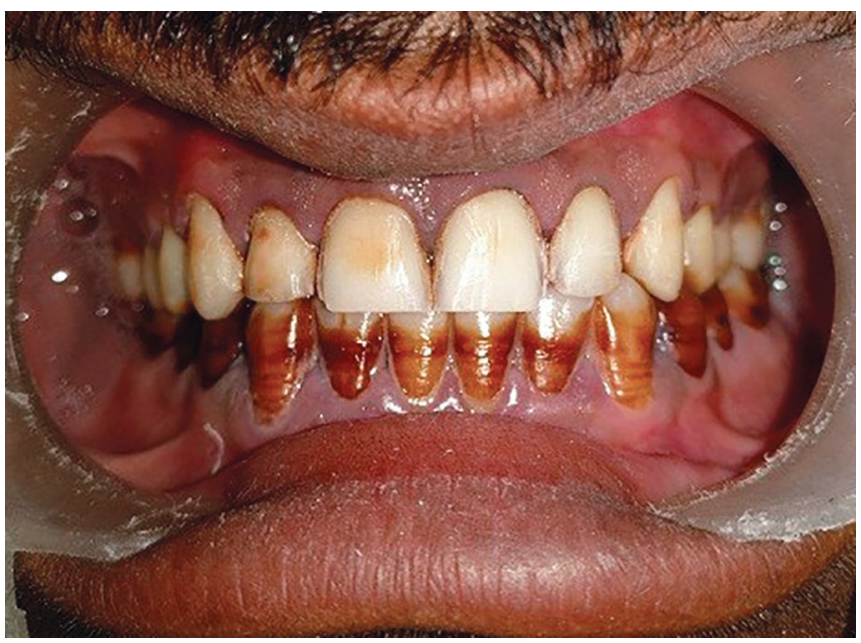

Fig. 4: Prepared maxillary teeth

grooves of $0.3 \mathrm{~mm}$ were placed in the cervical, middle, and incisal third of the teeth (Fig. 3). Remaining islands of unprepared enamel were reduced to the depth of the orientation grooves. Definitive chamfer margins (0.3-0.4 $\mathrm{mm}$ in depth) were then established using a long tapered medium diamond point (LVS3). The subgingival finish lines were carefully created parallel to the entire gingival margin from the distal papilla tip to the mesial papilla tip. The preparation was extended proximally both sides without breaking the contact point. The incisal edges were prepared as to provide maximum bulk for the porcelain with the wrap-around design. Therefore, an average of $1 \mathrm{~mm}$ incisal reduction was made with the finish line terminating lingually away from the contacts of the opposing teeth (Figs 4 and 5). After the teeth preparation was completed, chemico-mechanical gingival retraction was done using hemostatic agent (Gel Cord, Pascal International) and a gingival cord (Ultra Pak Dental Gingival Retraction Knitted Cord-00). Single-step impression (putty wash technique) was made using polyvinyl siloxane impression material. Temporization was 


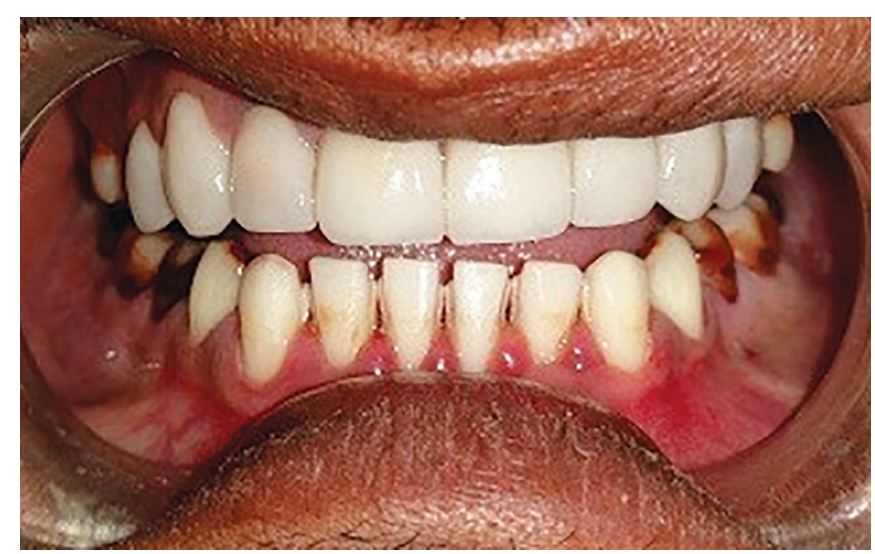

Fig. 5: Prepared mandibular teeth

not carried out since only 0.5 to $0.8 \mathrm{~mm}$ tooth reduction was performed.

\section{Fabrication of Porcelain Veneers}

Cast was poured with Type IV Gypsum Product (Kalrock) according to the manufacturer's recommendations. Stone dies were carefully separated from the impressions and two coats of die spacer were applied $0.5 \mathrm{~mm}$ short of the finish line of the preparations. The patterns were waxed-up on the dies, and veneers were fabricated from lithium disilicate-reinforced glass ceramic material (IPS E-max) using the heat press technique according to the manufacturer's recommendations.

\section{Try-in of Porcelain Veneers}

The correct fit of the veneers was verified individually and collectively first on the working model. The teeth surfaces were first cleaned with slurry of fine pumice and each porcelain veneer was individually tried using trial paste (glycerin) as the temporary luting medium. The order followed was central incisors first followed by the canines, premolars, and finally the lateral incisors. The adaptation and margin of each veneer was critically evaluated, and the interferences in eccentric movements were eliminated. Once patient was satisfied with the form, shape, and shade, the porcelain veneers were finished and glazed.

\section{Cementation of Porcelain Veneers}

The intaglio surface of porcelain veneers was acid-etched with $9 \%$ hydrofluoric acid, then followed by silanization. A silane coupling agent (Monobond S) was applied to the inner veneer surface and left for 60 seconds before airdrying. Gingival retraction cord was placed on the prepared teeth to decrease the crevicular fluid flow during cementation. Each tooth was etched with $37 \%$ phosphoric acid for 15 seconds then rinsed thoroughly and air-dried gently. Bonding application was done by scrubbing the

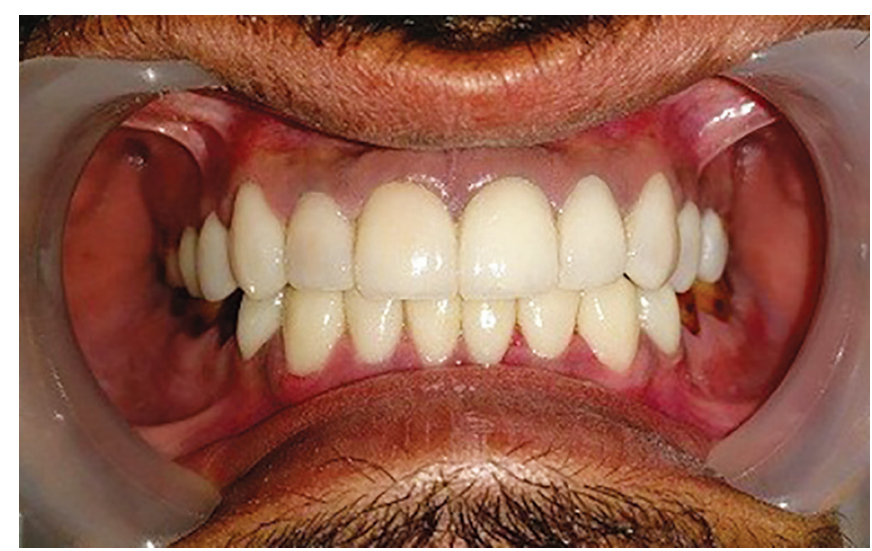

Fig. 6: Postoperative-maxillary and mandibular veneers

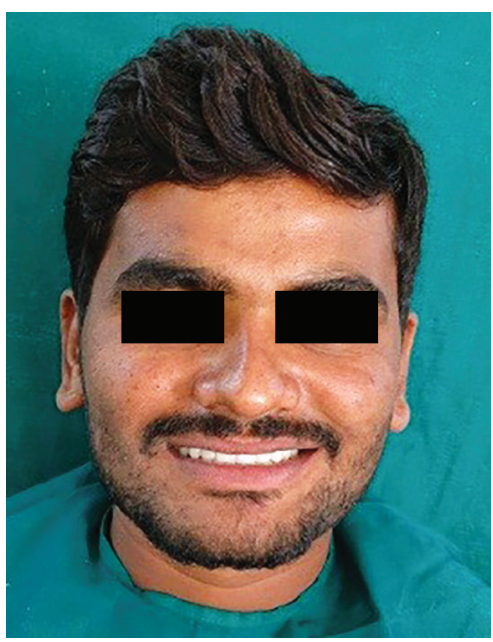

Fig. 7: Posttreatment frontal view

bonding agent (Universal) on each tooth and light curing it for 30 seconds. This was then followed by the application of a thin layer of light polymerizing composite resin luting cement (Variolink N LC, Ivoclar Vivadent) on the inner surface of the veneers. The veneer with the cement was carefully placed onto the prepared teeth, flash curing was carried out, and all excess cement was removed. Complete light polymerization was then done for 40 seconds from palatal, labial, and incisal sides, and the marginal area was finished and polished with abrasive disks. Centric and eccentric occlusal interferences were checked. Then the same steps were followed for the mandibular teeth maintaining the original occlusion of the patient. The contacts and occlusion were rechecked after the cementation of the mandibular veneers (Figs 6 and 7). Postoperative instructions regarding oral hygiene and avoidance of habits causing trauma to veneered teeth were given.

\section{DISCUSSION}

Dental fluorosis is a disturbance affecting the enamel formation during the time of mineralization stage. 
The clinical appearance reflects a spectrum of change. Lusterless white lines or diffuse opacities are present in its mild form, while in the more severe forms generalized opaque and chalky appearance with confluent pitting and staining of hypomineralized tissue may be seen. ${ }^{12}$ The process occurs initially with fluorides being absorbed in the stomach and small intestine, and then largely excreted via the kidneys. Any ingested fluoride is stored in the mineralized tissues, such as bone and teeth. Fluoride in excess during the tooth formative stage damages ameloblast cell product and retards the enamel matrix from calcifying properly. ${ }^{13}$

The treatment of enamel fluorosis usually ranges from ceramic veneer, free-hand bonding restorations to minimally invasive microabrasion techniques; it is the severity of the lesion that determines the treatment option. ${ }^{3,7,8}$ Other treatment options available are laser-assisted bleaching, abrasion employing abrasive pastes, and even combination of various treatment modalities. ${ }^{14,15}$ A newer alternative is restoring in a single visit with indirect, prefabricated composite veneers called Componeers (Coltène Whaledent). ${ }^{16}$ However, porcelain veneers are still considered the ultimate option for conservative esthetic treatment because they leave nearly all of the enamel intact before the veneer is placed. ${ }^{17}$ Ceramic veneers not only reduce the destructive approach and minimize the gingival reaction risk of full crowns but also mimic the translucency of natural tooth structure and can provide more promising esthetic results. Several clinical studies have reported the esthetic performance, biocompatibility, and durability of porcelain laminate veneers, their success rate has been clinically evaluated and has shown longevity of more than 10 to 15 years. Very few failures were reported due to debonding, microleakage, fracture, and caries. The incidence of irreparable failure was $7 \%$ or less in all of these longitudinal studies. ${ }^{18}$

However, veneers are not applicable to all severities of dental fluorosis. Minor fluorosis may not warrant the invasive removal of tooth structure, ${ }^{7}$ while porcelain veneers may not suffice to mask the stains of a severely fluorosed dentition. Indications should be observed accurately, and tooth tissue preservation must be given importance. In the present case, veneers were used, as the diagnosis was made as moderate fluorosis. Pleasing results were achieved where the patient was satisfied with the final outcome. A 3-month follow-up after treatment showed all restorations were fixed in place, esthetics was very good with sound margins and with no staining or discoloration.

\section{CONCLUSION}

Dental fluorosis is one of the major health issues in India with over 6 million children seriously affected. With the wide range of treatment modalities that are available, each case should be considered with caution and the most suitable treatment option should be selected. In this case, veneers were the most appropriate choice and gave high esthetic results. At the end of the treatment, the patient was extremely satisfied and pleased with the results.

\section{REFERENCES}

1. McKay FS, Black GV. Investigation of mottled teeth: An endemic imperfection of the enamel of teeth heretofore unknown in literature of dentistry. Dent Cosmos 1916;58: 129-156.

2. Fejerskov O, Kidd AM. Dental caries the disease and its management. Clinical use of fluoride. 1st ed. UK: Blackwell Munksgaard; 2003. pp. 189-202.

3. Nazirkar G, Meshram S. An evaluation of two modern allceramic crowns and their comparison with metal ceramic crowns in terms of the translucency and fracture strength. Int J Dent Clin 2011;3(1):5-7.

4. Chattopadhyay A. Oral health epidemiology: principles and practice. Burlington (MA): Jones \& Bartlett; 2010. p. 319.

5. Rajendran R. Shafer's textbook of oral pathology. 6th ed. India: Elsevier India; 2009. pp. 52-53.

6. Bussadori SK, do Rego MA, da Silva PE, Pinto MM, Pinto AC. Esthetic alternative for fluorosis blemishes with the usage of a dual bleaching system based on hydrogen peroxide at $35 \%$. J Clin Pediatr Dent 2004 Winter;28(2):143-146.

7. Benbachir N, Ardu S, Krejci I. Indications and limits of the microabrasion technique. Quintessence Int 2007 Nov-Dec;38(10):811-815.

8. Anand IS. Fluorosis varied treatment options. J Conserv Dent 2010 Jan;13(1):47-53.

9. Shortt WE. Endemic fluorosis in Nellore District, South India. Indian Med Gazette 1937 Jul;72(7):396-398.

10. Handa BK. Geochemistry and genesis of fluoride containing ground waters in India. Ground Water 1975;13:275-281.

11. Susheela AK. Fluorosis management programme in India. Curr Sci 1999 Nov;77(10).

12. Fejerskov O, Johnson N, Silverstone L. The ultrastructure of fluorosed human dental enamel. Eur J Oral Sci 1974;82(5): 357-372.

13. McCauley LK, Somerman MJ. Mineralized tissues in oral and craniofacial science: biological principles and clinical correlates. UK: John Wiley \& Sons; 2012. p. 338.

14. Ardu S, Stayridakis M, Krejci I. A minimally invasive treatment of severe dental fluorosis. Quintessence Int 2007 Jun;38(6);455-458.

15. Ng F, Manton DJ. Aesthetic management of severely fluorosed incisors in an adolescent female. Aust Dent J 2007 Sep;52(3):243-248.

16. Du Toit J, Patel N, Montalli V, Jain S. Aesthetic treatment of severely florosed teeth with prefabricated composite veneers: a case report. Int Dent 2012;2(6):44-50.

17. Javaheri D. Considerations for planning esthetic treatment with veneers involving no or minimal preparation. J Am Dent Assoc 2007 Mar 31;138(3):331-337.

18. Suha T, Kivanc U. Esthetic rehabilitation of crowded maxillary anterior teeth utilizing ceramic veneers: a case report. Cases J 2009;2(1):8329. 